\title{
Bayesian Inference of a Finite Mixture of Inverse Weibull Distributions with an Application to Doubly Censoring Data
}

\author{
Navid Feroze \\ AIOU, Pakistan \\ navidferoz@hotmail.com
}

\begin{abstract}
The families of mixture distributions have a wider range of applications in different fields such as fisheries, agriculture, botany, economics, medicine, psychology, electrophoresis, finance, communication theory, geology and zoology. They provide the necessary flexibility to model failure distributions of components with multiple failure modes. Mostly, the Bayesian procedure for the estimation of parameters of mixture model is described under the scheme of Type-I censoring. In particular, the Bayesian analysis for the mixture models under doubly censored samples has not been considered in the literature yet. The main objective of this paper is to develop the Bayes estimation of the inverse Weibull mixture distributions under doubly censoring. The posterior estimation has been conducted under the assumption of gamma and inverse levy using precautionary loss function and weighted squared error loss function. The comparisons among the different estimators have been made based on analysis of simulated and real life data sets.
\end{abstract}

Keywords: Inverse Transformation Method, Mixture Model, Doubly Censoring, Loss Functions, Bayes Estimator.

\section{Introduction}

In survival analysis, data are subject to censoring. The most common type of censoring is right censoring, in which the survival time is larger than the observed right censoring time. In some cases, however, data are subject to left, as well as, right censoring. When left censoring occurs, the only information available to an analyst is that the survival time is less than or equal to the observed left censoring time. A more complex censoring scheme is found when both initial and final times are interval-censored. This situation is referred as double censoring, or the data with both right and left censored observations are known as doubly censored data.

Analysis of doubly censored data for simple (single) distribution has been studied by many authors. Fernandez (2000) investigated maximum likelihood prediction based on type II doubly censored exponential data. Fernandez (2006) has discussed Bayesian estimation based on trimmed samples from Pareto populations. Khan et al. (2010) studied predictive inference from a two-parameter Rayleigh life model given a doubly censored sample. Kim and Song (2010) have discussed Bayesian estimation of the parameters of the generalized exponential distribution from doubly censored samples. Khan et al. (2011) studied sensitivity analysis of predictive modeling for responses from the threeparameter Weibull model with a follow-up doubly censored sample of cancer patients. Pak et al. (2013) has proposed the estimation of Rayleigh scale parameter under doubly type-II censoring from imprecise data.

In statistics, a mixture distribution is signified as a convex fusion of other probability distributions. It can be used to model a statistical population with subpopulations, where constituent of mixture probability densities are the densities of the subpopulations. 
Mixture distribution may appropriately be used for certain data set where the subsets of the whole data set possess different properties that can best be modeled separately. They can be more mathematically manageable, because the individual mixture components are dealt with more ease than the overall mixture density. The families of mixture distributions have a wider range of applications in different fields such as fisheries, agriculture, botany, economics, medicine, psychology, electrophoresis, finance, communication theory, geology and zoology.

Soliman (2006) derived estimators for the finite mixture of Rayleigh model based on progressively censored data. Sultan et al. (2007) have discussed some properties of the mixture of two inverse Weibull distributions. Saleem and Aslam (2008) presented a comparison of the Maximum Likelihood (ML) estimates with the Bayes estimates assuming the Uniform and the Jeffreys priors for the parameters of the Rayleigh mixture. Kundu and Howalder (2010) considered the Bayesian inference and prediction of the inverse Weibull distribution for type-II censored data. Saleem et al. (2010) considered the Bayesian analysis of the mixture of Power function distribution using the complete and the censored sample. Shi and Yan (2010) studied the case of the two parameter exponential distribution under type I censoring to get empirical Bayes estimates. Eluebaly and Bouguila (2011) have presented a Bayesian approach to analyze finite generalized Gaussian mixture models which incorporate several standard mixtures, widely used in signal and image processing applications, such as Laplace and Gaussian. Sultan and Moisheer (2012) developed approximate Bayes estimation of the parameters and reliability function of mixture of two inverse Weibull distributions under Type-2 censoring.

The article is outlined as follows. In section 2, we define the mixture model, sampling and likelihood function of Rayleigh model. In section 3, the posterior distributions are derived under different priors. Expressions for the said estimators and corresponding posterior risks are derived in the section 4. Elicitation of hyperparameters via prior predictive approach is discussed in the section 5. Simulation study and comparison of the estimates are given in the section 6. Real data set to illustrate the methodology of the proposed mixture model are discussed in the section 7. Some concluding remarks close the paper.

\section{The Model and Likelihood Function}

If the probability density function (pdf) of the Weibull distribution is:

$$
f_{i}\left(y_{i j}, \theta, \tau\right)=\theta_{i} \tau_{i} y_{i j}^{\theta_{i}-1} \exp \left(-\tau_{i} y_{i j}^{\theta_{i}}\right), y_{i j}>0, \quad i=1,2 \text {, and } j=1,2, \ldots, n_{i}
$$

Then the random variable $x_{i j}=1 / y_{i j}$ has the inverse Weibull distribution with pdf

$$
f_{i}\left(x_{i j}, \theta, \tau\right)=\theta_{i} \tau_{i} x_{i j}{ }^{-\left(\theta_{i}+1\right)} \exp \left(-\tau_{i} x_{i j}{ }^{-\theta_{i}}\right), x_{i j}>0 i=1,2 \text {, and } j=1,2, \ldots, n_{i}
$$

where $\theta_{i}>0$, and $\tau_{i}>0$ are shape and scale parameters respectively. 
The cumulative distribution function (CDF) of the distribution is:

$$
F_{i}\left(x_{i j}, \theta, \tau\right)=\exp \left(-\tau_{i} x_{i j}{ }^{-\theta_{i}}\right), x_{i j}>0, \theta_{i}>0 \text {, and } \tau_{i}>0 i=1,2 \text {, and } j=1,2, \ldots, n_{i}
$$

A density function for mixture of two components densities with mixing weights $\left(p_{1}\right.$, 1- $p_{1}$ )

$$
f(x)=p_{1} f_{1}(x)+\left(1-p_{1}\right) f_{2}(x), \quad 0<p_{1}<1
$$

The cumulative distribution function for the mixture model is:

$$
F(x)=p_{1} F_{1}(x)+\left(1-p_{1}\right) F_{2}(x)
$$

Consider a random sample of size ' $n$ ' from inverse Weibull distribution, and let $x_{r}, x_{r+1}, \ldots, x_{s}$ be the ordered observations that can only be observed. The remaining ' $r-1$ ' smallest observations and the ' $n-s$ ' largest observations have been assumed to be censored. Now based on causes of failure, the failed items are assumed to come either from subpopulation 1 or from subpopulation 2 ; so the $x_{1 r_{1}}, \ldots, x_{1 s_{1}}$ and $x_{2 r_{2}}, \ldots, x_{2 s_{2}}$ failed items come from first and second subpopulations respectively. The rest of the observations which are less than $x_{r}$ and greater than $x_{s}$ have been assumed to be censored from each component. Where $x_{s}=\max \left(x_{1, s_{1}}, x_{2, s_{2}}\right)$ and $x_{r}=\min \left(x_{1, r_{1}}, x_{2, r_{2}}\right)$. Therefore, $m_{1}=s_{1}-r_{1}+1$ and $m_{2}=s_{2}-r_{2}+1$ number of failed items can be observed from first and second subpopulation respectively. The remaining $n-(s-r+2)$ items are assumed to be censored observations, and $s-r+2$ are the uncensored items. Where $r=r_{1}+r_{2}, s=s_{1}+s_{2}$ and $m=m_{1}+m_{2}$. Then the likelihood function for the Type II doubly censored sample $\mathrm{x}=\left\{\left(x_{1 r_{1}}, \ldots, x_{1 s_{1}}\right),\left(x_{2 r_{2}}, \ldots, x_{2 s_{2}}\right)\right\}$, assuming the causes of the failure of the left censored items are identified, can be written as:

$$
\begin{aligned}
L\left(\tau_{1}, \tau_{2}, p_{1} \mid \mathrm{x}\right) \propto & \left\{F_{1}\left(x_{\left(r_{1}\right)}, \tau_{1}\right)\right\}^{r_{1}-1}\left\{F\left(x_{\left(r_{2}\right)}, \tau_{2}\right)\right\}^{r_{2}-1}\left\{1-F\left(x_{s}, \tau_{1}, \tau_{2}\right)\right\}^{n-s} \\
& \times\left\{\prod_{i=r_{1}}^{s_{1}} p_{1} f_{1}\left(x_{1(i)}, \tau_{1}\right)\right\}\left\{\prod_{i=r_{2}}^{s_{2}}\left(1-p_{1}\right) f_{2}\left(x_{2(i)}, \tau_{2}\right)\right\} \\
L\left(\tau_{1}, \tau_{2}, p_{1} \mid \mathrm{x}\right) \propto & \left\{\exp \left(-\tau_{1} x_{(\eta)}{ }^{-\theta_{1}}\right)\right\}^{r_{1}-1}\left\{\exp \left(-\tau_{2} x_{((2)}{ }^{-\theta_{2}}\right)\right\}^{r_{2}-1}\left\{1-p_{1} \exp \left(-\tau_{1} x_{(\eta)}{ }^{-\theta_{1}}\right)-p_{2} \exp \left(-\tau_{2} x_{(2)}{ }^{-\theta_{2}}\right)\right\}^{n-s} \\
& \times\left\{\prod_{i=r_{1}}^{s_{1}} p_{1} \theta_{1} \tau_{1} x_{1(i)}{ }^{-\left(\theta_{1}+1\right)} \exp \left(-\tau_{1} x_{1(i)}{ }^{-\theta_{1}}\right)\right\}\left\{\prod_{i=r_{2}}^{s_{2}}\left(1-p_{1}\right) \theta_{2} \tau_{2} x_{2(i)}{ }^{-\left(\theta_{2}+1\right)} \exp \left(-\tau_{2} x_{2(i)}{ }^{-\theta_{2}}\right)\right\}
\end{aligned}
$$

Assuming the shape parameter to be known the likelihood function (6) reduces

$$
\begin{aligned}
L\left(\tau_{1}, \tau_{2}, p_{1} \mid \mathrm{X}\right) \propto & \sum_{k 1=0}^{n-s} \sum_{k_{2}=0}^{k 1}(-1)^{k_{1}}\left(\begin{array}{c}
n-s \\
k_{1}
\end{array}\right)\left(\begin{array}{c}
k_{1} \\
k_{2}
\end{array}\right) p_{1}^{m_{1}+k_{1}-k_{2}}\left(1-p_{1}\right)^{m_{2}+k_{2}} \\
& \times \tau_{1}^{{ }^{m_{1}}} \tau_{2}^{{ }^{m_{2}}} \exp \left\{-\tau_{1}\left(\gamma_{1}\left(x_{1 j}\right)\right)\right\} \exp \left\{-\tau_{2}\left(\gamma_{2}\left(x_{2 j}\right)\right)\right\}
\end{aligned}
$$


where $\gamma_{1}\left(x_{1 j}\right)=\sum_{i=r_{1}}^{s_{1}} x_{1(i)}{ }^{-\theta_{1}}+\left(k_{1}-k_{2}\right) x_{(s)}{ }^{-\theta_{1}}+\left(r_{1}-1\right) x_{\left(r_{1}\right)}^{-\theta_{1}}, \quad \gamma_{2}\left(x_{2 j}\right)=\sum_{i=r_{2}}^{s_{2}} x_{2(i)}{ }^{-\theta_{1}}+\left(k_{2}\right) x_{(s)}{ }^{-\theta_{2}}+\left(r_{2}-1\right) x_{\left(r_{2}\right)}^{-\theta_{2}}$, $m_{1}=s_{1}-r_{1}+1$, and $m_{2}=s_{2}-r_{2}+1$

\section{Bayes Estimation}

The simple estimation of the scale parameter often pre-assumes the knowhow of the shape parameter (for more detail, see Panaitescu et al. (2010), Zanakis (2007), Kundu and Howaldar (2010), Shi and Yan (2010), etc.). For the Bayesian estimation, let us assume that the parameters $\tau_{i}$ and $p_{1} i=1,2$ are independent random variables, and then we consider the following priors for different parameters:

\subsection{Bayesian Estimation using Conjugate Prior}

The prior for the rate parameters $\tau_{i}$ for $i=1,2$, is assumed to be the gamma distribution, with the hyperparameters $a_{\mathrm{i}}$ and $b_{\mathrm{i}}$, given by

$$
f_{\tau_{i}}\left(\tau_{i}\right)=\frac{b_{i}^{a_{i}}}{\Gamma\left(a_{i}\right)} \tau_{i}^{a i-1} \exp \left(-\tau_{i} b_{i}\right), \quad a_{i}, b_{i}>0
$$

The prior for $p_{1}$ is the beta distribution, whose density is given by

$$
f_{p}\left(p_{1}\right)=\frac{\Gamma\left(c_{1}+d_{1}\right)}{\Gamma\left(c_{1}\right) \Gamma\left(d_{1}\right)} p_{1}^{c_{1}-1}\left(1-p_{1}\right)^{d_{1}-1}, \quad c_{1}, d_{1}>0
$$

From equation (8)-(9), we propose the following joint prior density of the vector $\Theta=\left(\tau_{1}, \tau_{2}, p_{1}\right)$ :

$$
g(\Theta) \propto \tau_{i}^{a i-1} \exp \left(-\tau_{i} b_{i}\right) p_{1}^{c_{1}-1}\left(1-p_{1}\right)^{d_{1}-1}, 0<p_{1}<1, a_{i}>0, b_{i}>0, c_{1}>0, d_{1}>0
$$

By multiplying equation (10) with equation (7), the joint posterior density for the vector $\Theta$ given the data becomes

$$
\begin{aligned}
& \pi(\Theta \mid \mathrm{x})=\Omega_{1}^{-1} \sum_{k=0}^{n-s} \sum_{k_{2}=0}^{k 1}(-1)^{k_{1}}\left(\begin{array}{c}
n-s \\
k_{1}
\end{array}\right)\left(\begin{array}{l}
k_{1} \\
k_{2}
\end{array}\right) p_{1}^{m_{1}+k_{1}-k_{2}+c_{1}-1}\left(1-p_{1}\right)^{m_{2}+k_{2}+d_{1}-1} \prod_{i=1}^{2} \tau_{i}^{\left(a_{i}+m_{i}\right)-1} \exp \left\{-\tau_{i}\left(\xi_{i}\left(x_{i j}\right)\right)\right\} \text { (11) } \\
& \text { where } \Omega_{1}=\sum_{k 1=0}^{n-s} \sum_{k_{2}=0}^{k 1}(-1)^{k_{1}}\left(\begin{array}{c}
n-s \\
k_{1}
\end{array}\right)\left(\begin{array}{l}
k_{1} \\
k_{2}
\end{array}\right) \operatorname{Beta}\left(m_{1}+k_{1}-k_{2}+c_{1}, m_{2}+k_{2}+d_{1}\right) \prod_{i=1}^{2} \frac{\Gamma\left(a_{i}+m_{i}\right)}{\left\{\xi_{i}\left(x_{i j}\right)\right\}^{\left(a_{i}+m_{i}\right)}}, \\
& \xi_{1}\left(x_{1 j}\right)=\gamma_{1}\left(x_{1 j}\right)+b_{1} \text { and } \xi_{2}\left(x_{2 j}\right)=\gamma_{2}\left(x_{2 j}\right)+b_{2}
\end{aligned}
$$

Marginal distributions of $\tau_{i}$ and $p_{1} i=1,2$ can be obtained by integrating the nuisance parameters. 


\subsection{Bayesian Estimation using Inverse Levy Prior}

The prior for the rate parameters $\tau_{i}$ for $i=1,2$, is assumed to be the inverse Levy distribution, with the hyperparameter $v_{\mathrm{i}}$, given by

$$
f_{\tau_{i}}\left(\tau_{i}\right)=\sqrt{\frac{\nu_{i}}{2 \pi}} \tau_{i}^{-1 / 2} \exp \left(\frac{-\tau_{i} v_{i}}{2}\right), \quad v_{i}>0
$$

The prior for $p_{1}$ is the beta distribution, whose density is given by

$$
f_{p}\left(p_{1}\right)=\frac{\Gamma\left(c_{2}+d_{2}\right)}{\Gamma\left(c_{2}\right) \Gamma\left(d_{2}\right)} p_{1}^{c_{2}-1}\left(1-p_{1}\right)^{d_{2}-1}, \quad c_{2}, d_{2}>0
$$

From equation (12)-(13), we propose the following joint prior density of the vector $\Theta=\left(\tau_{1}, \tau_{2}, p_{1}\right)$ :

$$
g(\Theta) \propto \tau_{i}^{-1 / 2} \exp \left(\frac{-\tau_{i} v_{i}}{2}\right) p_{1}^{c_{2}-1}\left(1-p_{1}\right)^{d_{2}-1}, 0<p_{1}<1, v_{i}>0, c_{2}>0, d_{2}>0
$$

By multiplying equation (14) with equation (7), the joint posterior density for the vector $\Theta$ given the data becomes

$$
\begin{aligned}
& \pi(\Theta \mid \mathrm{x})=\Omega_{2}^{-1} \sum_{k=0}^{n-s} \sum_{k_{2}=0}^{k 1}(-1)^{k_{1}}\left(\begin{array}{c}
n-s \\
k_{1}
\end{array}\right)\left(\begin{array}{c}
k_{1} \\
k_{2}
\end{array}\right) p_{1}^{m_{1}+k_{1}-k_{2}+c_{2}-1}\left(1-p_{1}\right)^{m_{2}+k_{2}+d_{2}-1} \prod_{i=1}^{2} \tau_{i}^{m_{i}-1 / 2} \exp \left\{-\tau_{i}\left(\psi_{i}\left(x_{i j}\right)\right)\right\} \text { (15) } \\
& \text { where } \Omega_{2}=\sum_{k 1=0}^{n-s} \sum_{k_{2}=0}^{k 1}(-1)^{k_{1}}\left(\begin{array}{c}
n-s \\
k_{1}
\end{array}\right)\left(\begin{array}{c}
k_{1} \\
k_{2}
\end{array}\right) \operatorname{Beta}\left(m_{1}+k_{1}-k_{2}+c_{2}, m_{2}+k_{2}+d_{2}\right) \prod_{i=1}^{2} \frac{\Gamma\left(m_{i}+1 / 2\right)}{\left\{\psi_{i}\left(x_{i j}\right)\right\}^{\left(m_{i}+1 / 2\right)}}, \\
& \psi_{1}\left(x_{1 j}\right)=\gamma_{1}\left(x_{1 j}\right)+v_{1} / 2 \text { and } \psi_{2}\left(x_{2 j}\right)=\gamma_{2}\left(x_{2 j}\right)+v_{2} / 2
\end{aligned}
$$

Marginal distributions of $\tau_{i}$ and $p_{1} i=1,2$ can be obtained by integrating the nuisance parameters.

\section{Bayes Estimation of the Vector of Parameters $\Theta$}

The Bayesian point estimation is connected to a loss function in general, signifying the loss is induced when the estimate $\hat{\theta}$ differ from true parameter $\theta$. It is often noticed that in some situations Bayes estimate under another loss function works better than the Bayes estimate under the true loss function, when true loss function exists. Since there is no specific rule that helps us to identify the appropriate loss function to be used. Precautionary loss function (PLF), which is defined as $l(\hat{\theta}, \theta)=(\theta-\hat{\theta})^{2} / \hat{\theta}$ is proposed by Norstrom (1996). Bayes estimator and posterior risk under (PLF) are $\hat{\theta}=\left\{E\left(\theta^{2} \mid \mathbf{x}\right)\right\}^{1 / 2} \quad$ and $\quad \rho(\hat{\theta})=2\left[\left\{E\left(\theta^{2} \mid \mathbf{x}\right)\right\}^{1 / 2}-E(\theta \mid \mathbf{x})\right] \quad$ respectively. 
generalization of the squared error loss which is of concern is $l(\hat{\theta}, \theta)=(\theta-\hat{\theta})^{2} / \theta$ called weighted squared error loss function. Bayes estimator and posterior risk under (WSELF) are $\hat{\theta}=\left\{E\left(\theta^{-1} \mid \mathbf{x}\right)\right\}^{-1}$ and $\rho(\hat{\theta})=E(\theta \mid \mathbf{x})-\left\{E\left(\theta^{-1} \mid \mathbf{x}\right)\right\}^{-1}$ respectively.

In this section, the respective marginal distribution of each parameter has been used to derive the Bayes estimators and posterior risks of $\tau_{1}, \tau_{2}$ and $p_{1}$ under precautionary loss function (PLF) and weighted squared error loss function (WSELF). The Bayes estimators and their posterior risks of the parameters $\tau_{1}, \tau_{2}$ and $p_{1}$ for the conjugate (gamma and beta) priors using the PLF and WSELF functions are given in this section.

The Bayes estimators of $\tau_{1}, \tau_{2}$ and $p_{1}$ under PLF using gamma prior are:

$$
\begin{aligned}
& \hat{\tau}_{1(\mathrm{PLF})}=\left(\frac{\sum_{k=0}^{n-s} \sum_{k_{2}=0}^{k 1}(-1)^{k_{1}}\left(\begin{array}{c}
n-s \\
k_{1}
\end{array}\right)\left(\begin{array}{l}
k_{1} \\
k_{2}
\end{array}\right) \operatorname{Beta}\left(m_{1}+k_{1}-k_{2}+c_{1}, m_{2}+k_{2}+d_{1}\right) \frac{\Gamma\left(a_{1}+m_{1}+2\right) \Gamma\left(a_{2}+m_{2}\right)}{\left\{b_{1}+\gamma_{1}\left(x_{1 j}\right)\right\}^{\left(a_{1}+m_{1}+2\right)}\left\{b_{2}+\gamma_{2}\left(x_{2 j}\right)\right\}^{\left(a_{2}+m_{2}\right)}}}{\sum_{k 1=0}^{n-s} \sum_{k_{2}=0}^{k 1}(-1)^{k_{1}}\left(\begin{array}{c}
n-s \\
k_{1}
\end{array}\right)\left(\begin{array}{l}
k_{1} \\
k_{2}
\end{array}\right) \operatorname{Beta}\left(m_{1}+k_{1}-k_{2}+c_{1}, m_{2}+k_{2}+d_{1}\right) \frac{\Gamma\left(a_{1}+m_{1}\right) \Gamma\left(a_{2}+m_{2}\right)}{\left\{b_{1}+\gamma_{1}\left(x_{1 j}\right)\right\}^{\left(a_{1}+m_{1}\right)}\left\{b_{2}+\gamma_{2}\left(x_{2 j}\right)\right\}^{\left(a_{2}+m_{2}\right)}}}\right)^{\frac{1}{2}} \\
& \hat{\tau}_{2(\mathrm{PLF})}=\left(\frac{\sum_{k=0}^{n-s} \sum_{k_{2}=0}^{k 1}(-1)^{k_{1}}\left(\begin{array}{c}
n-s \\
k_{1}
\end{array}\right)\left(\begin{array}{l}
k_{1} \\
k_{2}
\end{array}\right) \operatorname{Beta}\left(m_{1}+k_{1}-k_{2}+c_{1}, m_{2}+k_{2}+d_{1}\right) \frac{\Gamma\left(a_{1}+m_{1}\right) \Gamma\left(a_{2}+m_{2}+2\right)}{\left\{b_{1}+\gamma_{1}\left(x_{1 j}\right)\right\}^{\left(a_{1}+m_{1}\right)}\left\{b_{2}+\gamma_{2}\left(x_{2 j}\right)\right\}^{\left(a_{2}+m_{2}+2\right)}}}{\sum_{k=0}^{n-s} \sum_{k_{2}=0}^{k 1}(-1)^{k_{1}}\left(\begin{array}{c}
n-s \\
k_{1}
\end{array}\right)\left(\begin{array}{l}
k_{1} \\
k_{2}
\end{array}\right) \operatorname{Beta}\left(m_{1}+k_{1}-k_{2}+c_{1}, m_{2}+k_{2}+d_{1}\right) \frac{\Gamma\left(a_{1}+m_{1}\right) \Gamma\left(a_{2}+m_{2}\right)}{\left\{b_{1}+\gamma_{1}\left(x_{1 j}\right)\right\}^{\left(a_{1}+m_{1}\right)}\left\{b_{2}+\gamma_{2}\left(x_{2 j}\right)\right\}^{\left(a_{2}+m_{2}\right)}}}\right)^{\frac{1}{2}} \\
& \hat{p}_{1(\mathrm{PLF})}=\left(\frac{\sum_{k=0}^{n-s} \sum_{k_{2}=0}^{k 1}(-1)^{k_{1}}\left(\begin{array}{c}
n-s \\
k_{1}
\end{array}\right)\left(\begin{array}{c}
k_{1} \\
k_{2}
\end{array}\right) \operatorname{Beta}\left(m_{1}+k_{1}-k_{2}+c_{1}+2, m_{2}+k_{2}+d_{1}\right) \prod_{i=1}^{2} \frac{\Gamma\left(a_{i}+m_{i}\right)}{\left\{b_{i}+\gamma_{i}\left(x_{i j}\right)\right\}^{\left(a_{i}+m_{i}\right)}}}{\sum_{k 1=0}^{n-s} \sum_{k_{2}=0}^{k 1}(-1)^{k_{1}}\left(\begin{array}{c}
n-s \\
k_{1}
\end{array}\right)\left(\begin{array}{c}
k_{1} \\
k_{2}
\end{array}\right) \operatorname{Beta}\left(m_{1}+k_{1}-k_{2}+c_{1}, m_{2}+k_{2}+d_{1}\right) \prod_{i=1}^{2} \frac{\Gamma\left(a_{i}+m_{i}\right)}{\left\{b_{i}+\gamma_{i}\left(x_{i j}\right)\right\}^{\left(a_{i}+m_{i}\right)}}}\right)^{\frac{1}{2}}
\end{aligned}
$$

The posterior risks of $\tau_{1}, \tau_{2}$ and $p_{1}$ under PLF using gamma prior are:

$$
\begin{aligned}
& \rho\left(\hat{\tau}_{1(\mathrm{PLF})}\right)=2\left(\begin{array}{l}
\left\{\Omega_{1}^{-1} \sum_{k=0}^{n-s} \sum_{k_{2}=0}^{k 1}(-1)^{k_{1}}\left(\begin{array}{c}
n-s \\
k_{1}
\end{array}\right)\left(\begin{array}{l}
k_{1} \\
k_{2}
\end{array}\right) \operatorname{Beta}\left(m_{1}+k_{1}-k_{2}+c_{1}, m_{2}+k_{2}+d_{1}\right) \frac{\Gamma\left(a_{1}+m_{1}+2\right) \Gamma\left(a_{2}+m_{2}\right)}{\left\{b_{1}+\gamma_{1}\left(x_{1 j}\right)\right\}^{\left(a_{1}+m_{1}+2\right)}\left\{b_{2}+\gamma_{2}\left(x_{2 j}\right)\right\}^{\left(a_{2}+m_{2}\right)}}\right\}^{1 / 2} \\
-\Omega_{1}^{-1} \sum_{k=0}^{n-s} \sum_{k_{2}=0}^{k 1}(-1)^{k_{1}}\left(\begin{array}{c}
n-s \\
k_{1}
\end{array}\right)\left(\begin{array}{l}
k_{1} \\
k_{2}
\end{array}\right) \operatorname{Beta}\left(m_{1}+k_{1}-k_{2}+c_{1}, m_{2}+k_{2}+d_{1}\right) \frac{\Gamma\left(a_{1}+m_{1}+1\right) \Gamma\left(a_{2}+m_{2}\right)}{\left\{b_{1}+\gamma_{1}\left(x_{1 j}\right)\right\}^{\left(a_{1}+m_{1}+1\right)}\left\{b_{2}+\gamma_{2}\left(x_{2 j}\right)\right\}^{\left(a_{2}+m_{2}\right)}}
\end{array}\right) \\
& \rho\left(\hat{\tau}_{2}(\mathrm{PLF})\right)=2\left(\begin{array}{l}
\left\{\Omega_{1}^{-1} \sum_{k=0}^{n-s} \sum_{k_{2}=0}^{k 1}(-1)^{k_{1}}\left(\begin{array}{c}
n-s \\
k_{1}
\end{array}\right)\left(\begin{array}{l}
k_{1} \\
k_{2}
\end{array}\right) \operatorname{Beta}\left(m_{1}+k_{1}-k_{2}+c_{1}, m_{2}+k_{2}+d_{1}\right) \frac{\Gamma\left(a_{1}+m_{1}\right) \Gamma\left(a_{2}+m_{2}+2\right)}{\left\{b_{1}+\gamma_{1}\left(x_{1 j}\right)\right\}^{\left(a_{1}+m_{1}\right)}\left\{b_{2}+\gamma_{2}\left(x_{2 j}\right)\right\}^{\left(a_{2}+m_{2}+2\right)}}\right\}^{1 / 2} \\
-\Omega_{1}^{-1} \sum_{k=0}^{n-s} \sum_{k_{2}=0}^{k 1}(-1)^{k_{1}}\left(\begin{array}{c}
n-s \\
k_{1}
\end{array}\right)\left(\begin{array}{l}
k_{1} \\
k_{2}
\end{array}\right) \operatorname{Beta}\left(m_{1}+k_{1}-k_{2}+c_{1}, m_{2}+k_{2}+d_{1}\right) \frac{\Gamma\left(a_{1}+m_{1}\right) \Gamma\left(a_{2}+m_{2}+1\right)}{\left\{b_{1}+\gamma_{1}\left(x_{1 j}\right)\right\}^{\left(a_{1}+m_{1}\right)}\left\{b_{2}+\gamma_{2}\left(x_{2 j}\right)\right\}^{\left(a_{2}+m_{2}+1\right)}}
\end{array}\right)
\end{aligned}
$$


Bayesian Inference of a Finite Mixture of Inverse Weibull Distributions with an Application to Doubly .....

$$
\rho\left(\hat{p}_{1} \quad(\mathrm{PLF})\right)=2\left(\begin{array}{l}
\left\{\Omega_{1}^{-1} \sum_{k=0}^{n-s} \sum_{k_{2}=0}^{k 1}(-1)^{k_{1}}\left(\begin{array}{c}
n-s \\
k_{1}
\end{array}\right)\left(\begin{array}{l}
k_{1} \\
k_{2}
\end{array}\right) \operatorname{Beta}\left(m_{1}+k_{1}-k_{2}+c_{1}+2, m_{2}+k_{2}+d_{1}\right) \prod_{i=1}^{2} \frac{\Gamma\left(a_{i}+m_{i}\right)}{\left\{b_{i}+\gamma_{i}\left(x_{i j}\right)\right\}^{\left(a_{i}+m_{i}\right)}}\right\}^{1 / 2} \\
-\Omega_{1}^{-1} \sum_{k 1=0}^{n-s} \sum_{k_{2}=0}^{k 1}(-1)^{k_{1}}\left(\begin{array}{c}
n-s \\
k_{1}
\end{array}\right)\left(\begin{array}{c}
k_{1} \\
k_{2}
\end{array}\right) \operatorname{Beta}\left(m_{1}+k_{1}-k_{2}+c_{1}+1, m_{2}+k_{2}+d_{1}\right) \prod_{i=1}^{2} \frac{\Gamma\left(a_{i}+m_{i}\right)}{\left\{b_{i}+\gamma_{i}\left(x_{i j}\right)\right\}^{\left(a_{i}+m_{i}\right)}}
\end{array}\right)
$$

The Bayes estimators of $\tau_{1}, \tau_{2}$ and $p_{1}$ under WSELF using gamma prior are:

$$
\begin{aligned}
& \hat{\tau}_{1 \text { (WSELF) }}=\left(\frac{\sum_{k=0}^{n-s} \sum_{k_{2}=0}^{k 1}(-1)^{k_{1}}\left(\begin{array}{c}
n-s \\
k_{1}
\end{array}\right)\left(\begin{array}{l}
k_{1} \\
k_{2}
\end{array}\right) \operatorname{Beta}\left(m_{1}+k_{1}-k_{2}+c_{1}, m_{2}+k_{2}+d_{1}\right) \frac{\Gamma\left(a_{1}+m_{1}-1\right) \Gamma\left(a_{2}+m_{2}\right)}{\left\{b_{1}+\gamma_{1}\left(x_{1 j}\right)\right\}^{\left(a_{1}+m_{1}-1\right)}\left\{b_{2}+\gamma_{2}\left(x_{2 j}\right)\right\}^{\left(a_{2}+m_{2}\right)}}}{\sum_{k 1=0}^{n-s} \sum_{k_{2}=0}^{k 1}(-1)^{k_{1}}\left(\begin{array}{c}
n-s \\
k_{1}
\end{array}\right)\left(\begin{array}{c}
k_{1} \\
k_{2}
\end{array}\right) \operatorname{Beta}\left(m_{1}+k_{1}-k_{2}+c_{1}, m_{2}+k_{2}+d_{1}\right) \frac{\Gamma\left(a_{1}+m_{1}\right) \Gamma\left(a_{2}+m_{2}\right)}{\left\{b_{1}+\gamma_{1}\left(x_{1 j}\right)\right\}^{\left(a_{1}+m_{1}\right)}\left\{b_{2}+\gamma_{2}\left(x_{2 j}\right)\right\}^{\left(a_{2}+m_{2}\right)}}}\right)^{-1} \\
& \hat{\tau}_{2} \text { (WSELF) }=\left(\frac{\sum_{k=0}^{n-s} \sum_{k_{2}=0}^{k 1}(-1)^{k_{1}}\left(\begin{array}{c}
n-s \\
k_{1}
\end{array}\right)\left(\begin{array}{l}
k_{1} \\
k_{2}
\end{array}\right) \operatorname{Beta}\left(m_{1}+k_{1}-k_{2}+c_{1}, m_{2}+k_{2}+d_{1}\right) \frac{\Gamma\left(a_{1}+m_{1}\right) \Gamma\left(a_{2}+m_{2}-1\right)}{\left\{b_{1}+\gamma_{1}\left(x_{1 j}\right)\right\}^{\left(a_{1}+m_{1}\right)}\left\{b_{2}+\gamma_{2}\left(x_{2 j}\right)\right\}^{\left(a_{2}+m_{2}-1\right)}}}{\sum_{k=0}^{n-s} \sum_{k_{2}=0}^{k 1}(-1)^{k_{1}}\left(\begin{array}{c}
n-s \\
k_{1}
\end{array}\right)\left(\begin{array}{l}
k_{1} \\
k_{2}
\end{array}\right) \operatorname{Beta}\left(m_{1}+k_{1}-k_{2}+c_{1}, m_{2}+k_{2}+d_{1}\right) \frac{\Gamma\left(a_{1}+m_{1}\right) \Gamma\left(a_{2}+m_{2}\right)}{\left\{b_{1}+\gamma_{1}\left(x_{1 j}\right)\right\}^{\left(a_{1}+m_{1}\right)}\left\{b_{2}+\gamma_{2}\left(x_{2 j}\right)\right\}^{\left(a_{2}+m_{2}\right)}}}\right)^{-1} \\
& \hat{p}_{1 \text { (WSELF) }}=\left(\frac{\sum_{k 1=0}^{n-s} \sum_{k_{2}=0}^{k 1}(-1)^{k_{1}}\left(\begin{array}{c}
n-s \\
k_{1}
\end{array}\right)\left(\begin{array}{l}
k_{1} \\
k_{2}
\end{array}\right) \operatorname{Beta}\left(m_{1}+k_{1}-k_{2}+c_{1}-1, m_{2}+k_{2}+d_{1}\right) \prod_{i=1}^{2} \frac{\Gamma\left(a_{i}+m_{i}\right)}{\left\{b_{i}+\gamma_{i}\left(x_{i j}\right)\right\}^{\left(a_{i}+m_{i}\right)}}}{\sum_{k 1=0}^{n-s} \sum_{k_{2}=0}^{k 1}(-1)^{k_{1}}\left(\begin{array}{c}
n-s \\
k_{1}
\end{array}\right)\left(\begin{array}{l}
k_{1} \\
k_{2}
\end{array}\right) \operatorname{Beta}\left(m_{1}+k_{1}-k_{2}+c_{1}, m_{2}+k_{2}+d_{1}\right) \prod_{i=1}^{2} \frac{\Gamma\left(a_{i}+m_{i}\right)}{\left\{b_{i}+\gamma_{i}\left(x_{i j}\right)\right\}^{\left(a_{i}+m_{i}\right)}}}\right)^{-1}
\end{aligned}
$$

The posterior risks of $\tau_{1}, \tau_{2}$ and $p_{1}$ using gamma prior are:

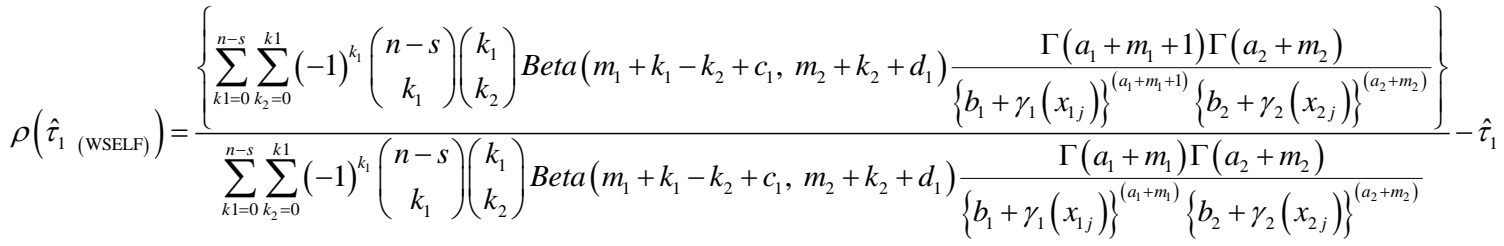

$$
\begin{aligned}
& \rho\left(\hat{\tau}_{2} \text { (WSELF) }\right)=\frac{\left\{\sum_{k=0}^{n-s} \sum_{k_{2}=0}^{k 1}(-1)^{k_{1}}\left(\begin{array}{c}
n-s \\
k_{1}
\end{array}\right)\left(\begin{array}{l}
k_{1} \\
k_{2}
\end{array}\right) \operatorname{Beta}\left(m_{1}+k_{1}-k_{2}+c_{1}, m_{2}+k_{2}+d_{1}\right) \frac{\Gamma\left(a_{1}+m_{1}\right) \Gamma\left(a_{2}+m_{2}+1\right)}{\left.\left\{b_{1}+\gamma_{1}\left(x_{1 j}\right)\right\}^{\left(a_{1}+m_{1}\right)}\left\{b_{2}+\gamma_{2}\left(x_{2 j}\right)\right\}^{\left(a_{2}+m_{2}+1\right)}\right\}}\right\}}{\sum_{k=0}^{n-s} \sum_{k_{2}=0}^{k 1}(-1)^{k_{1}}\left(\begin{array}{c}
n-s \\
k_{1}
\end{array}\right)\left(\begin{array}{l}
k_{1} \\
k_{2}
\end{array}\right) \operatorname{Beta}\left(m_{1}+k_{1}-k_{2}+c_{1}, m_{2}+k_{2}+d_{1}\right) \frac{\Gamma\left(a_{1}+m_{1}\right) \Gamma\left(a_{2}+m_{2}\right)}{\left\{b_{1}+\gamma_{1}\left(x_{1 j}\right)\right\}^{\left(a_{1}+m_{1}\right)}\left\{b_{2}+\gamma_{2}\left(x_{2 j}\right)\right\}^{\left(a_{2}+m_{2}\right)}}}-\hat{\tau}_{2} \\
& \rho\left(\hat{p}_{1}(\mathrm{wSELF})\right)=\frac{\left\{\sum_{k 1=0}^{n-s} \sum_{k_{2}=0}^{k 1}(-1)^{k_{1}}\left(\begin{array}{c}
n-s \\
k_{1}
\end{array}\right)\left(\begin{array}{c}
k_{1} \\
k_{2}
\end{array}\right) \operatorname{Beta}\left(m_{1}+k_{1}-k_{2}+c_{1}+1, m_{2}+k_{2}+d_{1}\right) \prod_{i=1}^{2} \frac{\Gamma\left(a_{i}+m_{i}\right)}{\left.\left\{b_{i}+\gamma_{i}\left(x_{i j}\right)\right\}^{\left(a_{i}+m_{i}\right)}\right\}}\right.}{\sum_{k=0}^{n-s} \sum_{k_{2}=0}^{k 1}(-1)^{k_{1}}\left(\begin{array}{c}
n-s \\
k_{1}
\end{array}\right)\left(\begin{array}{c}
k_{1} \\
k_{2}
\end{array}\right) \operatorname{Beta}\left(m_{1}+k_{1}-k_{2}+c_{1}, m_{2}+k_{2}+d_{1}\right) \prod_{i=1}^{2} \frac{\Gamma\left(a_{i}+m_{i}\right)}{\left\{b_{i}+\gamma_{i}\left(x_{i j}\right)\right\}^{\left(a_{i}+m_{i}\right)}}}-\hat{p}_{1}
\end{aligned}
$$

Thus, expressions for Bayes estimators and their posterior risks under the inverse Levy can be obtained with little alteration. 


\section{Elicitation}

In Bayesian analysis the elicitation of opinion is a crucial step. It helps to make it easy for us to understand what the experts believe in, and what their opinions are. In statistical inference, the characteristics of a certain predictive distribution proposed by an expert determine the hyperparameters of a prior distribution. In this article, we focused on a method of elicitation based on prior predictive distribution. The elicitation of hyperparameter from the prior $p(\lambda)$ is a difficult task. The prior predictive distribution is used for the elicitation of the hyperparameters which is compared with the experts' judgment about this distribution and then the hyperparameters are chosen in such a way so as to make the judgment agree closely as possible with the given distribution. Readers desiring more detail may refer to: Grimshaw et al. (2001), O'Hagan et al. (2006), Jenkinson (2005) and Leon et al. (2003). According to Aslam (2003), the method of elicitation is to compare the prior predictive distribution with experts' assessment about this distribution and then to choose the hyperparameters that make the assessment agree closely with the member of the family. The prior predictive distributions under all the priors are derived using the following formula:

$$
p(y)=\int_{\Theta} p(y \mid \Theta) p(\Theta) d \Theta
$$

\subsection{Elicitation under gamma distribution}

The prior predictive distribution using gamma prior is:

$$
p(y)=\frac{\theta_{1} y^{-\left(\theta_{1}+1\right)} a_{1} b_{1}^{a_{1}} c_{1}}{\left(c_{1}+d_{1}\right)\left(y^{-\theta_{1}}+b_{1}\right)^{\left(a_{1}+1\right)}}+\frac{\theta_{2} y^{-\left(\theta_{2}+1\right)} a_{2} b_{2}{ }^{a_{2}} d_{1}}{\left(c_{1}+d_{1}\right)\left(y^{-\theta_{2}}+b_{2}\right)^{\left(a_{2}+1\right)}}, y>0
$$

We have assumed $\left(\theta_{1}, \theta_{2}\right)=(1,1)$ for convenience in calculations. For the elicitation of the six hyperparameters, six different intervals are considered. From equation (16), the experts' probabilities/assessments are supposed to be 0.10 for each case. The six integrals for equation (19) are considered with the following limits of the values of random variable ' $Y^{\prime}$ : $(0,10),(10,20),(20,30),(30,40),(40,50)$ and $(50,60)$ respectively. For the elicitation of hyperparameters, $a_{1}, a_{2}, b_{1}, b_{2}, c_{1}$, and $d_{1}$. these six integrals are solved simultaneously through computer program developed in $S A S$ package using the command of PROC SYSLIN. Thus the values of hyperparameters obtained by applying this methodology are: 4.982587, 3.356211,0.987542, 0.46523, 1.45987 and 0.05690.

\subsection{Elicitation under Inverse Levy Prior}

$$
p(y)=\frac{\theta_{1} y^{-\left(\theta_{1}+1\right)}\left(v_{1} / 2\right)^{1 / 2} c_{1}}{2\left(c_{1}+d_{1}\right)\left(y^{-\theta_{1}}+v_{1} / 2\right)^{\left(v_{1} / 2+1\right)}}+\frac{\theta_{2} y^{-\left(\theta_{2}+1\right)}\left(v_{2} / 2\right)^{1 / 2} d_{2}}{2\left(c_{1}+d_{1}\right)\left(y^{-\theta_{2}}+v_{2} / 2\right)^{\left(v_{2} / 2+1\right)}}, y>0 .
$$


Now, we have to elicit four hyperparameters, so we have to consider the four integrals. The expert probabilities are assumed to 0.15 for each integral with the following limits of the values of random variable ' $Y$ ': $(0,15),(15,30),(30,45)$ and $(45,60)$. Using the similar kind of program, as discussed above, we have the following values of hyperparameters $v_{1}=0.062138, v_{2}=0.19136, c_{2}=0.895777$ and $d_{2}=0.63889$.

\section{Simulation Study and Comparisons}

This section consists of the simulation study to compare the performance of the discussed estimators on the basis of generated samples from the inverse Weibull mixture distribution using doubly censored data. We have assumed $\left(\theta_{1}, \theta_{2}\right)=(1,1)$ for convenience in calculations. We take random samples of sizes $n=20,40$, and 80 from the two component mixture of inverse Weibull distributions with following choice of parametric values $\quad\left(\tau_{1}, \tau_{2}\right) \in\{(0.1,0.15),(10,15),(0.1,15),(10,0.15)\}$, $p_{1}=0.45$ and 0.6. To develop a mixture data we adopt the probabilistic mixing with probability $p_{1}$ and $\left(1-p_{1}\right)$. A uniform number $u$ is generated $n$ times and if $u<p_{1}$ the observation is taken randomly from $F_{1}$ (the inverse Weibull distribution with parameter $\tau_{1}$ ) otherwise from $F_{2}$ (from the inverse Weibull with parameter $\tau_{2}$ ). Hence the parameters to be estimated are known to be $\left(\tau_{1}, \tau_{2}\right)$ and $p_{1}$. The choice of the censoring time is made in such a way that the censoring rate in the resultant sample is to be approximately $20 \%$. The simulated data sets have been obtained using following steps:

Step 1: Draw samples of size ' $n$ ' from the mixture model

Step 2: Generate a uniform random no. $u$ for each observation

Step 3: If $u \leq \pi$, the take the observation from first subpopulation otherwise from the second subpopulation

Step 4: Determine the test termination points on left and right, that is, determine the values of $x_{r}$ and $x_{s}$

Step 5: The observations which are less than $x_{r}$ and greater than $x_{s}$ have been considered to be censored from each component

Step 6: Use the remaining observations from each component for the analysis

To avoid an extreme sample, we simulate 10, 000 data sets each of size $n$. The Bayes estimates and posterior risks (in parenthesis) are computed using Mathematica 8.0. The average of these estimates and corresponding risks are reported in Tables 1-8. The abbreviations used in the tables are: B.Es: Bayes estimators; P.Rs: Posterior risks; GP: Gamma prior; ILP: Inverse Levy prior. 
Table 1: B.Es and their P.Rs under GP for

$$
\left(\tau_{1}, \tau_{2}, p_{1}\right)=(0.1,0.15,0.45) \text { and }(0.1,0.15,0.60)
$$

\begin{tabular}{|l|l|l|l|l|l|l|}
\hline \multirow{2}{*}{$n$} & \multicolumn{6}{l}{ Precautionary loss function } \\
\cline { 2 - 7 } & $\hat{\tau}_{1}$ & $\hat{\tau}_{2}$ & $\hat{p}_{1}$ & $\hat{\tau}_{1}$ & $\hat{\tau}_{2}$ & $\hat{p}_{1}$ \\
\hline \multirow{2}{*}{20} & 0.183998 & 0.210992 & 0.520207 & 0.157451 & 0.273239 & 0.670615 \\
& $(0.012870)$ & $(0.015336)$ & $(0.024894)$ & $(0.0080078)$ & $(0.025618)$ & $(0.017334)$ \\
\hline \multirow{2}{*}{40} & 0.141437 & 0.189734 & 0.476807 & 0.128095 & 0.19939 & 0.64533 \\
& $(0.006581)$ & $(0.008025)$ & $(0.014814)$ & $(0.004569)$ & $(0.011663)$ & $(0.010202)$ \\
\hline \multirow{2}{*}{80} & 0.121225 & 0.179241 & 0.459446 & 0.121049 & 0.181134 & 0.636047 \\
& $(0.004236)$ & $(0.005333)$ & $(0.010484)$ & $(0.003139)$ & $(0.007711)$ & $(0.007219)$ \\
\hline \multirow{2}{*}{20} & Weighted squared error loss function & & & \\
\hline \multirow{2}{*}{40} & 0.162952 & 0.19599 & 0.478401 & 0.141383 & 0.223633 & 0.643437 \\
& $(0.0130423)$ & $(0.016329)$ & $(0.028097)$ & $(0.008997)$ & $(0.025234)$ & $(0.019361)$ \\
\hline \multirow{2}{*}{80} & 0.12213 & 0.185997 & 0.45076 & 0.123577 & 0.18100 & 0.62999 \\
& $(0.006237)$ & $(0.008474)$ & $(0.015847)$ & $(0.004704)$ & $(0.011856)$ & $(0.010825)$ \\
\hline
\end{tabular}

Table 2: B.Es and their P.Rs under GP for $\left(\tau_{1}, \tau_{2}, p_{1}\right)=(10,15,0.45)$ and $(10,15,0.60)$

\begin{tabular}{|c|c|c|c|c|c|c|}
\hline \multirow{2}{*}{$n$} & \multicolumn{6}{|c|}{ Precautionary loss function } \\
\hline & $\hat{\tau}_{1}$ & $\hat{\tau}_{2}$ & $\hat{p}_{1}$ & $\hat{\tau}_{1}$ & $\hat{\tau}_{2}$ & $\hat{p}_{1}$ \\
\hline \multirow{2}{*}{20} & 7.918210 & 12.7604 & 0.512701 & 8.24576 & 12.0699 & 0.673449 \\
\hline & $(0.562497)$ & $(0.919622)$ & $(0.025237)$ & $(0.472256)$ & $(0.15019)$ & $(0.017232)$ \\
\hline \multirow{2}{*}{40} & 8.47102 & 14.0426 & 0.471625 & 8.95589 & 12.9464 & 0.64686 \\
\hline & $(0.398777)$ & $(0.589584)$ & $(0.014899)$ & $(0.319934)$ & $(0.765791)$ & $(0.010184)$ \\
\hline \multirow{2}{*}{80} & 8.90369 & 14.3116 & 0.45554 & 9.13910 & 13.33460 & 0.636388 \\
\hline & $(0.310527)$ & $(0.425427)$ & $(0.007971)$ & $(0.23731)$ & $(0.570857)$ & $(0.007250)$ \\
\hline \multicolumn{7}{|c|}{ Weighted squared error loss function } \\
\hline \multirow{2}{*}{20} & 6.95149 & 11.47690 & 0.471111 & 7.62418 & 9.97148 & 0.646635 \\
\hline & $(0.565598)$ & $(0.948828)$ & $(0.028409)$ & $(0.485881)$ & $(1.14909)$ & $(0.019255)$ \\
\hline \multirow{2}{*}{40} & 7.76927 & 13.06160 & 0.448141 & 8.56982 & 11.6106 & 0.631632 \\
\hline & $(0.399421)$ & $(0.59337)$ & $(0.015889)$ & $(0.326552)$ & $(0.769426)$ & $(0.010805)$ \\
\hline \multirow{2}{*}{80} & 8.26151 & 13.92270 & 0.448517 & 8.89651 & 12.71100 & 0.625797 \\
\hline & $(0.311853)$ & $(0.437513)$ & $(0.010896)$ & $(0.242402)$ & $(0.58988)$ & $(0.006540)$ \\
\hline
\end{tabular}


Bayesian Inference of a Finite Mixture of Inverse Weibull Distributions with an Application to Doubly .....

Table 3: B.Es and their P.Rs under GP for $\left(\tau_{1}, \tau_{2}, p_{1}\right)=(0.10,15,0.45)$ and $(0.10,15,0.60)$

\begin{tabular}{|l|l|l|l|l|l|l|}
\hline \multirow{2}{*}{$n$} & \multicolumn{6}{l}{ Precautionary loss function } \\
\cline { 2 - 7 } & $\hat{\tau}_{1}$ & $\hat{\tau}_{2}$ & $\hat{p}_{1}$ & $\hat{\tau}_{1}$ & $\hat{\tau}_{2}$ & $\hat{p}_{1}$ \\
\hline \multirow{2}{*}{20} & 0.16186 & 13.54830 & 0.474766 & 0.146025 & 13.1666 & 0.618725 \\
& $(0.011790)$ & $(0.900287)$ & $(0.024783)$ & $(0.008728)$ & $(1.09648)$ & $(0.018182)$ \\
\hline \multirow{4}{*}{0} & 0.127299 & 14.81350 & 0.435332 & 0.123062 & 14.7135 & 0.615899 \\
& $(0.00141)$ & $(0.568845)$ & $(0.014397)$ & $(0.004604)$ & $(0.716151)$ & $(0.010529)$ \\
\hline \multirow{2}{*}{80} & 0.117819 & 15.40490 & 0.452022 & 0.108965 & 15.34370 & 0.607914 \\
& $(0.004246)$ & $(0.4158504$ & $(0.009173)$ & $(0.003038)$ & $(0.540336)$ & $(0.007618)$ \\
\hline Weighted squared error loss function & & & & \\
\hline \multirow{2}{*}{20} & 0.147975 & 12.28820 & 0.434705 & 0.140810 & 11.39540 & 0.589610 \\
& $(0.012349)$ & $(0.923973)$ & $(0.027690)$ & $(0.009397)$ & $(1.11182)$ & $(0.020201)$ \\
\hline \multirow{2}{*}{40} & 0.118479 & 14.08820 & 0.44281 & 0.110857 & 13.14360 & 0.57350 \\
& $(0.006241)$ & $(0.579943)$ & $(0.015292)$ & $(0.004437)$ & $(0.720862)$ & $(0.011141)$ \\
\hline \multirow{2}{*}{80} & 0.114257 & 14.5437 & 0.450933 & 0.106203 & 14.29730 & 0.596316 \\
& $(0.0043927)$ & $(0.412396)$ & $(0.009751)$ & $(0.003032)$ & $(0.545823)$ & $(0.005546)$ \\
\hline
\end{tabular}

Table 4: B.Es and their P.Rs under GP for $\left(\tau_{1}, \tau_{2}, p_{1}\right)=(10,0.15,0.45)$ and $(10,0.15,0.60)$

\begin{tabular}{|l|l|l|l|l|l|l|}
\hline \multirow{2}{*}{$n$} & \multicolumn{6}{l}{ Precautionary loss function } \\
\cline { 2 - 7 } & $\hat{\tau}_{1}$ & $\hat{\tau}_{2}$ & $\hat{p}_{1}$ & $\hat{\tau}_{1}$ & $\hat{\tau}_{2}$ & $\hat{p}_{1}$ \\
\hline \multirow{2}{*}{20} & 8.16501 & 0.213878 & 0.566323 & 8.40326 & 0.252588 & 0.710226 \\
& $(0.522519)$ & $(0.016322)$ & $(0.020654)$ & $(0.450088)$ & $(0.025007)$ & $(0.013816)$ \\
\hline \multirow{2}{*}{40} & 9.53031 & 0.168253 & 0.53525 & 9.43799 & 0.171463 & 0.68998 \\
& $(0.387634)$ & $(0.007612)$ & $(0.0119353)$ & $(0.307876)$ & $(0.010648)$ & $(0.007560)$ \\
\hline \multirow{2}{*}{80} & 9.95986 & 0.158290 & 0.523602 & 9.80757 & 0.164939 & 0.62817 \\
& $(0.290613)$ & $(0.005088)$ & $(0.008327)$ & $(0.230131)$ & $(0.007261)$ & $(0.005086)$ \\
\hline Weighted squared error loss function & & & & \\
\hline \multirow{2}{*}{20} & 7.55109 & 0.19143 & 0.533040 & 8.13207 & 0.220316 & 0.688048 \\
& $(0.544131)$ & $(0.016855)$ & $(0.023020)$ & $(0.480553)$ & $(0.026363)$ & $(0.0153175)$ \\
\hline \multirow{2}{*}{40} & 8.80390 & 0.161305 & 0.516536 & 8.82344 & 0.170619 & 0.677469 \\
& $(0.385674)$ & $(0.007924)$ & $(0.012660)$ & $(0.305347)$ & $(0.0118843)$ & $(0.008418)$ \\
\hline \multirow{2}{*}{80} & 9.30670 & 0.153653 & 0.508710 & 9.64879 & 0.148905 & 0.670460 \\
& $(0.292979)$ & $(0.005234)$ & $(0.008488)$ & $(0.236021)$ & $(0.007314)$ & $(0.005437)$ \\
\hline
\end{tabular}


Table 5: B.Es and their P.Rs under ILP for $\left(\tau_{1}, \tau_{2}, p_{1}\right)=(0.1,0.15,0.45)$ and $(0.1,0.15,0.60)$

\begin{tabular}{|c|c|c|c|c|c|c|}
\hline \multirow{2}{*}{$n$} & \multicolumn{6}{|c|}{ Precautionary loss function } \\
\hline & $\hat{\tau}_{1}$ & $\hat{\tau}_{2}$ & $\hat{p}_{1}$ & $\hat{\tau}_{1}$ & $\hat{\tau}_{2}$ & $\hat{p}_{1}$ \\
\hline 20 & $\begin{array}{l}0.127691 \\
(0.013163)\end{array}$ & $\begin{array}{l}0.177611 \\
(0.016013)\end{array}$ & $\begin{array}{l}0.485151 \\
(0.026508)\end{array}$ & $\begin{array}{l}0.121899 \\
(0.009401)\end{array}$ & $\begin{array}{l}0.182759 \\
(0.023537)\end{array}$ & $\begin{array}{l}0.64281 \\
(0.018733)\end{array}$ \\
\hline 40 & $\begin{array}{l}0.110914 \\
(0.006591)\end{array}$ & $\begin{array}{l}0.172404 \\
(0.008203)\end{array}$ & $\begin{array}{l}0.456984 \\
(0.015296)\end{array}$ & $\begin{array}{l}0.113023 \\
(0.004813)\end{array}$ & $\begin{array}{l}0.168986 \\
(0.011884)\end{array}$ & $\begin{array}{l}0.62988 \\
(0.010635)\end{array}$ \\
\hline 80 & $\begin{array}{l}0.105183 \\
(0.004385)\end{array}$ & $\begin{array}{l}0.164297 \\
(0.005319)\end{array}$ & $\begin{array}{l}0.450695 \\
(0.010548)\end{array}$ & $\begin{array}{l}0.108526 \\
(0.003198)\end{array}$ & $\begin{array}{l}0.167578 \\
(0.008080)\end{array}$ & $\begin{array}{l}0.621729 \\
(0.006645)\end{array}$ \\
\hline \multicolumn{7}{|c|}{ Weighted squared error loss function } \\
\hline 20 & $\begin{array}{l}0.107436 \\
(0.013662)\end{array}$ & $\begin{array}{l}0.154796 \\
(0.016713)\end{array}$ & $\begin{array}{l}0.442314 \\
(0.029916)\end{array}$ & $\begin{array}{l}0.105422 \\
(0.009459)\end{array}$ & $\begin{array}{l}0.149031 \\
(0.025018)\end{array}$ & $\begin{array}{l}0.612117 \\
(0.021025)\end{array}$ \\
\hline 40 & $\begin{array}{l}0.101026 \\
(0.006722)\end{array}$ & $\begin{array}{l}0.153699 \\
(0.008510)\end{array}$ & $\begin{array}{l}0.443702 \\
(0.016316)\end{array}$ & $\begin{array}{l}0.102699 \\
(0.004750)\end{array}$ & $\begin{array}{l}0.15455 \\
(0.012422)\end{array}$ & $\begin{array}{l}0.61200 \\
(0.0113582)\end{array}$ \\
\hline 80 & $\begin{array}{l}0.097219 \\
(0.004383)\end{array}$ & $\begin{array}{l}0.152891 \\
(0.005444)\end{array}$ & $\begin{array}{l}0.449901 \\
(0.011171)\end{array}$ & $\begin{array}{l}0.099979 \\
(0.003115)\end{array}$ & $\begin{array}{l}0.152524 \\
(0.008062)\end{array}$ & $\begin{array}{l}0.601227 \\
(0.007708)\end{array}$ \\
\hline
\end{tabular}

Table 6: B.Es and their P.Rs under ILP for $\left(\tau_{1}, \tau_{2}, p_{1}\right)=(10,15,0.45)$ and $(10,15,0.60)$

\begin{tabular}{|c|c|c|c|c|c|c|}
\hline \multirow{2}{*}{$N$} & \multicolumn{6}{|c|}{ Precautionary loss function } \\
\hline & $\hat{\tau}_{1}$ & $\hat{\tau}_{2}$ & $\hat{p}_{1}$ & $\hat{\tau}_{1}$ & $\hat{\tau}_{2}$ & $\hat{p}_{1}$ \\
\hline 20 & $\begin{array}{l}11.54270 \\
(1.19393)\end{array}$ & $\begin{array}{l}15.78400 \\
(1.42804)\end{array}$ & $\begin{array}{l}0.486493 \\
(0.026501)\end{array}$ & $\begin{array}{l}11.48880 \\
(0.885081)\end{array}$ & $\begin{array}{l}16.38070 \\
(2.11917)\end{array}$ & $\begin{array}{l}0.644266 \\
(0.018660)\end{array}$ \\
\hline 40 & $\begin{array}{l}10.78201 \\
(0.638153)\end{array}$ & $\begin{array}{l}15.3402 \\
(0.733376)\end{array}$ & $\begin{array}{l}0.459242 \\
(0.015272)\end{array}$ & $\begin{array}{l}11.00650 \\
(0.467721)\end{array}$ & $\begin{array}{l}15.4734 \\
(1.09394)\end{array}$ & $\begin{array}{l}0.631273 \\
(0.10601)\end{array}$ \\
\hline 80 & $\begin{array}{l}10.5890 \\
(0.439570)\end{array}$ & $\begin{array}{l}15.14441 \\
(0.500679)\end{array}$ & $\begin{array}{l}0.457813 \\
(0.010707)\end{array}$ & $\begin{array}{l}10.77321 \\
(0.316332)\end{array}$ & $\begin{array}{l}15.4127 \\
(0.748079)\end{array}$ & $\begin{array}{l}0.625869 \\
(0.007298)\end{array}$ \\
\hline \multicolumn{7}{|c|}{ Weighted squared error loss function } \\
\hline 20 & $\begin{array}{l}9.6918 \\
(1.23064)\end{array}$ & $\begin{array}{l}13.8270 \\
(1.492460)\end{array}$ & $\begin{array}{l}0.442583 \\
(0.029893)\end{array}$ & $\begin{array}{l}10.222580 \\
(0.91153)\end{array}$ & $\begin{array}{l}12.33190 \\
(2.094820)\end{array}$ & $\begin{array}{l}0.61656 \\
(0.020826)\end{array}$ \\
\hline 40 & $\begin{array}{l}9.70297 \\
(0.645132)\end{array}$ & $\begin{array}{l}14.77460 \\
(0.772058)\end{array}$ & $\begin{array}{l}0.443817 \\
(0.016319)\end{array}$ & $\begin{array}{l}10.1601 \\
(0.466147)\end{array}$ & $\begin{array}{l}13.83130 \\
(1.04578)\end{array}$ & $\begin{array}{l}0.610646 \\
(0.011236)\end{array}$ \\
\hline 80 & $\begin{array}{l}9.93870 \\
(0.44666)\end{array}$ & $\begin{array}{l}15.07190 \\
(0.519686)\end{array}$ & $\begin{array}{l}0.447321 \\
(0.011155)\end{array}$ & $\begin{array}{l}10.10782 \\
(0.316407)\end{array}$ & $\begin{array}{l}14.57050 \\
(0.775175)\end{array}$ & $\begin{array}{l}0.610372 \\
(0.007727)\end{array}$ \\
\hline
\end{tabular}


Bayesian Inference of a Finite Mixture of Inverse Weibull Distributions with an Application to Doubly .....

Table 7: B.Es and their P.Rs under ILP for $\left(\tau_{1}, \tau_{2}, p_{1}\right)=(0.10,15,0.45)$ and $(0.10,15,0.60)$

\begin{tabular}{|c|c|c|c|c|c|c|}
\hline \multirow{2}{*}{$n$} & \multicolumn{6}{|c|}{ Precautionary loss function } \\
\hline & $\hat{\tau}_{1}$ & $\hat{\tau}_{2}$ & $\hat{p}_{1}$ & $\hat{\tau}_{1}$ & $\hat{\tau}_{2}$ & $\hat{p}_{1}$ \\
\hline 20 & $\begin{array}{l}0.113891 \\
(0.012322)\end{array}$ & $\begin{array}{l}17.90741 \\
(1.392810)\end{array}$ & $\begin{array}{l}0.446898 \\
(0.25974)\end{array}$ & $\begin{array}{l}0.116951 \\
(0.009551)\end{array}$ & $\begin{array}{l}18.10440 \\
(1.97170)\end{array}$ & $\begin{array}{l}0.590388 \\
(0.019419)\end{array}$ \\
\hline 40 & $\begin{array}{l}0.101979 \\
(0.006277)\end{array}$ & $\begin{array}{l}17.48260 \\
(0.753463)\end{array}$ & $\begin{array}{l}0.449381 \\
(0.014747)\end{array}$ & $\begin{array}{l}0.103028 \\
(0.004631)\end{array}$ & $\begin{array}{l}17.6810 \\
(1.030430)\end{array}$ & $\begin{array}{l}0.59747 \\
(0.010889)\end{array}$ \\
\hline 80 & $\begin{array}{l}0.094424 \\
(0.004061)\end{array}$ & $\begin{array}{l}16.38130 \\
(0.479399)\end{array}$ & $\begin{array}{l}0.451028 \\
(0.009569)\end{array}$ & $\begin{array}{l}0.097250 \\
(0.003015)\end{array}$ & $\begin{array}{l}16.74250 \\
(0.666457)\end{array}$ & $\begin{array}{l}0.59867 \\
(0.006751)\end{array}$ \\
\hline \multicolumn{7}{|c|}{ Weighted squared error loss function } \\
\hline 20 & $\begin{array}{l}0.090966 \\
(0.012129)\end{array}$ & $\begin{array}{l}14.83110 \\
(1.41690)\end{array}$ & $\begin{array}{l}0.404816 \\
(0.029059)\end{array}$ & $\begin{array}{l}0.101476 \\
(0.009664)\end{array}$ & $\begin{array}{l}14.44741 \\
(1.943110)\end{array}$ & $\begin{array}{l}0.559089 \\
(0.021586)\end{array}$ \\
\hline 40 & $\begin{array}{l}0.092145 \\
(0.006355)\end{array}$ & $\begin{array}{l}15.67300 \\
(0.730601)\end{array}$ & $\begin{array}{l}0.439736 \\
(0.015672)\end{array}$ & $\begin{array}{l}0.094058 \\
(0.004588)\end{array}$ & $\begin{array}{l}15.31430 \\
(0.993869)\end{array}$ & $\begin{array}{l}0.56776 \\
(0.011525)\end{array}$ \\
\hline 80 & $\begin{array}{l}0.096173 \\
(0.004214)\end{array}$ & $\begin{array}{l}15.660901 \\
(0.483135)\end{array}$ & $\begin{array}{l}0.454473 \\
(0.010071)\end{array}$ & $\begin{array}{l}0.098058 \\
(0.003196)\end{array}$ & $\begin{array}{l}15.10425 \\
(0.793825)\end{array}$ & $\begin{array}{l}0.58945 \\
(0.008256)\end{array}$ \\
\hline
\end{tabular}

Table 8: B.Es and their P.Rs under ILP for $\left(\tau_{1}, \tau_{2}, p_{1}\right)=(10,0.15,0.45)$ and $(10,0.15,0.60)$

\begin{tabular}{|l|l|l|l|l|l|l|}
\hline \multirow{2}{*}{$n$} & \multicolumn{6}{l}{ Precautionary loss function } \\
\cline { 2 - 7 } & $\hat{\tau}_{1}$ & $\hat{\tau}_{2}$ & $\hat{p}_{1}$ & $\hat{\tau}_{1}$ & $\hat{\tau}_{2}$ & $\hat{p}_{1}$ \\
\hline \multirow{2}{*}{20} & 12.51210 & 0.170129 & 0.539734 & 12.0588 & 0.184551 & 0.683249 \\
& $(1.120190)$ & $(0.016066)$ & $(0.021809)$ & $(0.848933)$ & $(0.025185)$ & $(0.015041)$ \\
\hline \multirow{2}{*}{40} & 11.90580 & 0.152507 & 0.520874 & 11.86440 & 0.150392 & 0.675412 \\
& $(0.581756)$ & $(0.007923)$ & $(0.012278)$ & $(0.433103)$ & $(0.011354)$ & $(0.008319)$ \\
\hline \multirow{2}{*}{80} & 11.78190 & 0.14776 & 0.513769 & 11.1487 & 0.150152 & 0.62718 \\
& $(0.405284)$ & $(0.005231)$ & $(0.008354)$ & $(0.290233)$ & $(0.007433)$ & $(0.005063)$ \\
\hline \multicolumn{7}{|l|}{ Weighted squared error loss function } \\
\hline \multirow{2}{*}{20} & 11.68720 & 0.1379633 & 0.504726 & 11.33731 & 0.138891 & 0.65924 \\
& $(1.239050)$ & $(0.016191)$ & $(0.024295)$ & $(0.909669)$ & $(0.025252)$ & $(0.016662)$ \\
\hline \multirow{2}{*}{40} & 10.91920 & 0.140533 & 0.501675 & 10.81830 & 0.142696 & 0.642486 \\
& $(0.594303)$ & $(0.008030)$ & $(0.013022)$ & $(0.442718)$ & $(0.011539)$ & $(0.008792)$ \\
\hline \multirow{2}{*}{80} & 10.26490 & 0.148138 & 0.49162 & 10.61130 & 0.15384 & 0.66391 \\
& $(0.412552)$ & $(0.005351)$ & $(0.007930)$ & $(0.291512)$ & $(0.007762)$ & $(0.005686)$ \\
\hline
\end{tabular}

The simulation study has revealed some interesting properties of the Bayes estimates. It is worth mentioning that in each case the posterior risks of estimates of lifetime parameters are decreasing as the sample size increases. The posterior risks of the estimates of $\tau_{1}, \tau_{2}$ have been assessed to be quite large when the values of the parameters are large and entirely small for rather smaller values of $\tau_{1}, \tau_{2}$. Another interesting point regarding the posterior risks of the estimates of parameters $\tau_{1}, \tau_{2}$ is that by increasing (decreasing) the 
proportion of the component in mixture reduces (increases) the posterior risk of the concerned $\tau$ parameter's estimate.

It has been observed that for the relatively smaller value of $\tau$ i.e. $(0.1,0.15)$, the performance of the precautionary loss function and the gamma prior is better than their counterparts, as the amounts of posterior risks are smaller than those in case of their counterparts. However, inverse Levy prior produces some closer estimates to the true value of parameters. Estimates of mixing proportion are found to be underestimated using inverse levy prior when $p_{1}=0.45$, but they are pretty good under gamma prior. When we consider the estimation of comparatively larger value of $\tau$ i.e. $(10,15)$, again under estimation is observed of the estimates of parameters under both priors and loss functions. But extent of under estimation is higher under precautionary loss function using gamma prior. Nonetheless, this underestimation is due to the random procedure and is tolerable. Further this problem can be faced off by using lager sample sizes. As far as the efficiency of the prior is concerned, gamma is found to be the efficient than inverse Levy prior. Moreover, on assessing the behavior of estimates, in case of the extremely different value of the parameters $\left(\tau_{1},<\tau_{2}\right.$ and $\left.\tau_{1}>\tau_{2}\right)=(0.1,15$ and $10,0.15)$ i.e. one is small and other is hundred fold large, it is noticed that the parameters are once again underestimated, and this underestimation is higher at every point using precautionary loss function under both priors. However, the use of weighted squared error loss function has exhibited the pretty good estimates with few exceptions, in terms of convergence. In general, the estimates under gamma prior using precautionary loss function are the best as the amounts of posterior risks associated with these estimates are the least in almost all the cases.

\section{Real Data Analysis}

In this section, we have analyzed real data sets to illustrate the methodology discussed in previous sections. In order to show the usefulness of the proposed mixture model, we applied the findings in this paper to the survival times (in days) of guinea pigs, injected with different doses of tubercle bacilli, in table 9. This data set has been discussed by Kundu and Howlader (2010). Singh (2013) has also analyzed this data set. The regimen number is the common logarithm of the number of bacillary units in $0.5 \mathrm{ml}$. of challenge solution; i.e., regimen 6.6 corresponds to $4.0 * 10^{6}$ bacillary units per $0.5 \mathrm{ml}$. Corresponding to regimen 6.6, there are 72 observations listed below. Further we used the Kolmogorov-Smirnov and chi square tests to see whether the data follow the inverse weibull distribution. These tests say that the data follow the inverse weibull distribution at $5 \%$ level of significance with p-values 0.1361 and 0.1290 respectively. We have assumed $\left(\theta_{1}, \theta_{2}\right)=(1,1)$ for convenience in calculations.

Table 9: Survival times (in days) of guinea pigs injected with different doses of tubercle bacilli

$12,15,22,24,24,32,32,33,34,38,38,43,44,48,52,53,54,54,55,56,57,58,58,59$, $60,60,60,60,61,62,63,65,65,67,68,70,70,72,73,75,76,76,81,83,84,85,87,91$, $95,96,98,99,109,110,121,127,129,131,143,146,146,175,175,211,233,258,258$, $263,297,341,341,376$ 
Now we consider the case when the data are doubly type II censored. Data are randomly grouped into two sets when $p_{1}=0.45$. It is assumed that we observe 33 data points belonging to population I and 39 data points belonging to population II respectively. To implement censored samplings, the $x_{1 r_{1}}, \ldots, x_{1 s_{1}}$ and $x_{2 r_{2}}, \ldots, x_{2 s_{2}}$ failed items come from first and second subpopulations respectively. The rest of the observations which are less than $x_{r}$ and greater than $x_{s}$ have been assumed to be censored from each component. Here, $m_{1}=s_{1}-r_{1}+1$ and $m_{2}=s_{2}-r_{2}+1$ number of failed items can be observed from first and second subpopulation respectively. The remaining $n-(s-r+2)$ items are assumed to be censored observations, and $s-r+2$ are the uncensored items. Where $r=r_{1}+r_{2}$, $s=s_{1}+s_{2}$ and $m=m_{1}+m_{2}$. The detail of the censored mixture data is as under:

Table 10: Doubly censored mixture real life data

\begin{tabular}{l|l}
\hline Population-I & Population-II \\
\hline $61,12,24,60,24,32,65,34,68,38$, & $15,131,87,143,91,95,175,110,121,127$, \\
$43,67,72,48,54,73,76,55,81,83,58$, & $297,341,60,62,65,63,70,, 96,211,98,258$, \\
$84,233,341,263,146,175,129,146$, & $258,70,75,76,59,60,57,56,58,53,54,44$, \\
$109,99,35,376$ & $52,43,38,33,32,22$ \\
\hline
\end{tabular}

The following characteristics are extracted from censored data for the analysis of mixture model:

$$
\begin{aligned}
& p_{1}=0.45, n=72, r=8, r_{1}=4, r_{2}=43, s=64, s_{1}=29, s_{2}=35, n_{1}=33, n_{2}=39, \\
& \theta_{1}=\theta_{2}=0.5, x_{r_{1}}=32, x_{s_{1}}=233, x_{r_{2}}=33, \text { and } x_{s_{2}}=258 . \\
& \sum_{i=r_{1}}^{s_{1}} x_{1(i)}^{-\theta_{1}}=3.21314 \text { and } \sum_{i=r_{2}}^{s_{2}} x_{2(i)}^{-\theta_{2}}=3.85409 .
\end{aligned}
$$

The similar methodology has been employed when $p_{1}=0.60, n=72, r=8, r_{1}=5, r_{2}=3$, $s=64, s_{1}=39, s_{2}=25, n_{1}=44, \quad n_{2}=28, \quad \theta_{1}=\theta_{2}=0.5$, $x_{r_{1}}=33, x_{s_{1}}=211, x_{r_{2}}=32$, and $x_{s_{2}}=175, \sum_{i=r_{1}}^{s_{1}} x_{1(i)}^{-\theta_{1}}=4.16450$ and $\sum_{i=r_{2}}^{s_{2}} x_{2(i)}^{-\theta_{2}}=3.21392$.

Bayes estimates are obtained assuming informative priors under minimum expected loss function, and k-loss function. 
Table 11: B.Es and their P.Rs under minimum expected loss function, and k-loss function for real data set

\begin{tabular}{|l|l|l|l|l|l|l|}
\hline \multirow{2}{*}{$\begin{array}{l}\text { Priors } \\
p_{1}=0.45\end{array}$} & \multicolumn{2}{l|}{ Precautionary loss function } & \multicolumn{2}{l|}{ Weighted squared error loss function } \\
\cline { 2 - 7 } & $\hat{\tau}_{1}$ & $\hat{\tau}_{2}$ & $\hat{p}_{1}$ & $\hat{\tau}_{1}$ & $\hat{\tau}_{2}$ & $\hat{p}_{1}$ \\
\hline Gamma Prior & 7.24033 & 8.12311 & 0.462888 & 6.916430 & 7.81032 & 0.449092 \\
& $(0.214571)$ & $(0.207398)$ & $(0.008964)$ & $(0.216613)$ & $(0.209089)$ & $(0.009315)$ \\
\hline \multirow{2}{*}{ Inverse Levy Prior } & 7.88449 & 8.14470 & 0.455393 & 7.47857 & 7.80556 & 0.441383 \\
& $(0.268576)$ & $(0.224745)$ & $(0.009102)$ & $(0.271625)$ & $(0.226764)$ & $(0.009460)$ \\
\hline \multirow{2}{*}{$p_{1}=0.60$} & $\hat{\lambda}_{1}$ & $\hat{\lambda}_{2}$ & $\hat{p}_{1}$ & $\hat{\lambda}_{1}$ & $\hat{\lambda}_{2}$ & $\hat{p}_{1}$ \\
\hline \multirow{2}{*}{ Gamma Prior } & 7.57257 & 7.23829 & 0.617057 & 7.31514 & 6.85812 & 0.607212 \\
& $(0.170820)$ & $(0.251558)$ & $(0.006404)$ & $(0.172019)$ & $(0.254391)$ & $(0.006642)$ \\
\hline \multirow{2}{*}{ Inverse Levy Prior } & 8.12849 & 7.17986 & 0.609343 & 7.82155 & $\begin{array}{l}6.76003 \\
(0.281074)\end{array}$ & $\begin{array}{l}0.599315 \\
(0.006767)\end{array}$ \\
\hline
\end{tabular}

The results in the table 11 indicate that the Bayes estimates under gamma prior are better than those under inverse levy prior under both loss functions. Similarly in comparison of the loss functions it has been assessed that the performance of the precautionary loss function is better than weighted squared error loss function. The larger values of the mixing parameter $\left(p_{1}\right)$ impose a positive impact on the performance of the estimation of the first component of the mixture. Hence the analysis of real life data indorsed the findings of the simulation study, suggesting the preference of gamma prior along with precautionary loss function.

\subsection{Graphical Representation of Posterior Risks under Different Loss Functions Various Priors}

Risks of the estimators are empirically evaluated based on a Monte-Carlo simulation study of samples. A number of values of unknown parameters are considered. Sample size is varied to observe the effect of small and large samples on the estimators. Different combinations of parameters are considered in studying the change in the estimators and their risks. The results are summarized in figures 1-4. It is easy to observe that the risk of the estimators will be a function of sample size, population parameters, hyperparameters of the prior distribution. After an extensive study of the results, the conclusions are drawn regarding the behavior of the estimators, which are summarized below. It may be mentioned here that because of space restrictions, all results are not shown in the graphs. It is noted that as sample size increases, the risk of all the estimators decrease, see figures 1-4. The effect of variation of parameters on the risks of the estimator has also been studied. It has been noticed that the risk of the estimators increases when increase the value of parameters. 


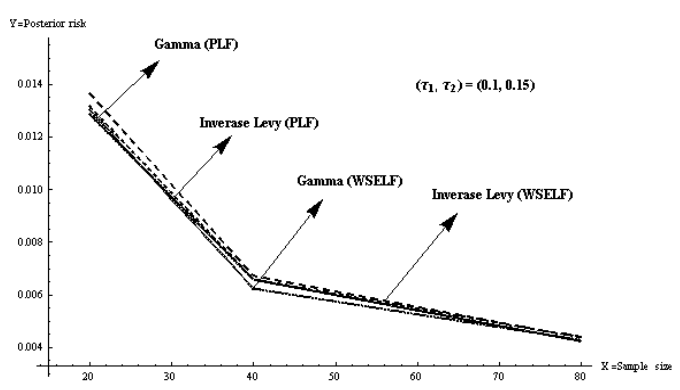

Fig.1 Posterior risks of $\tau_{1}$ for

$$
\left(\tau_{1}, \tau_{2}, p_{1}\right)=(0.10,0.15,0.45) \text {. }
$$

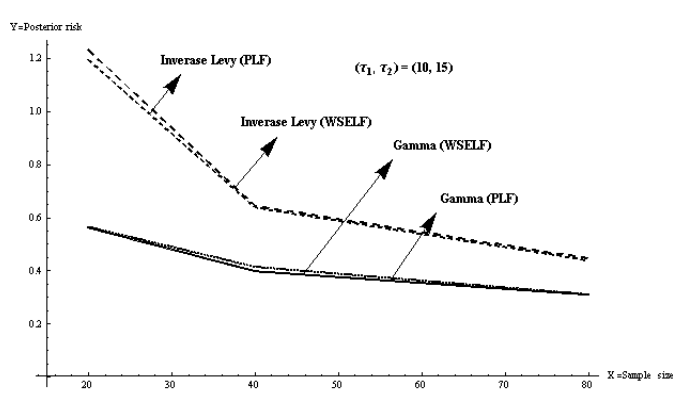

Fig.3 Posterior risks of $\tau_{1}$ for

$$
\left(\tau_{1}, \tau_{2}, p_{1}\right)=(10,15,0.45)
$$

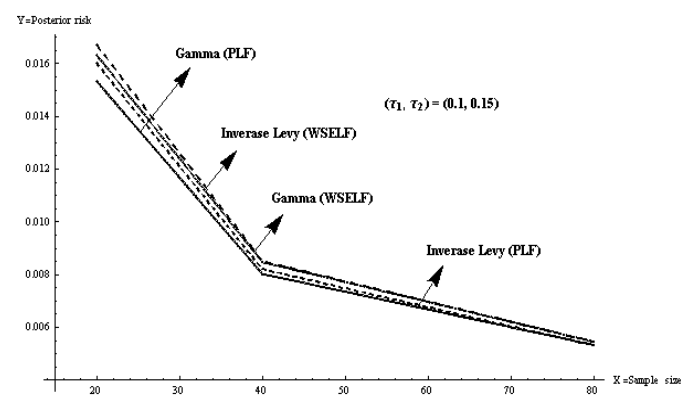

Fig.2 Posterior risks of $\tau_{2}$ for

$$
\left(\tau_{1}, \tau_{2}, p_{1}\right)=(0.10,0.15,0.45) \text {. }
$$

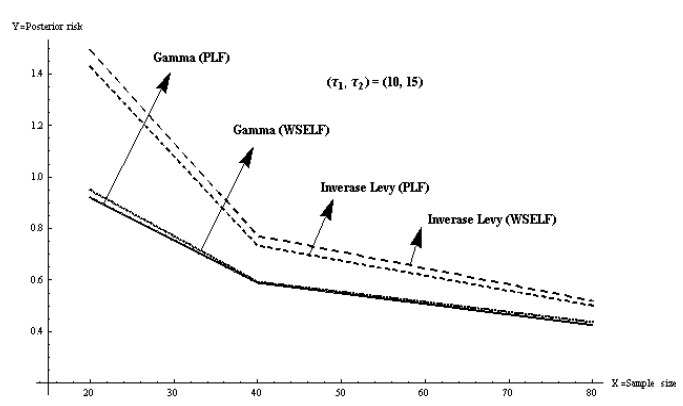

Fig.4 Posterior risks of $\tau_{2}$ for $\left(\tau_{1}, \tau_{2}, p_{1}\right)=(10,15,0.45)$

\section{Conclusion}

In this article, we have considered the Bayesian inference of inverse Weibull mixture distribution based on doubly type II censored data. The prior belief of the model is represented by the independent gamma, beta priors and inverse Levy, beta priors on the scale and mixing proportion parameters. Numerical results of the simulation study presented in tables $1-8$ exposed salient properties of the proposed Bayes estimators. The parameters of the mixture distributions have been over/under estimated under different cases. In general the larger values of the parameters have been over estimated and smaller values of the parameters have been under estimated in majority of the cases. However, it is nice to observe that the estimated values converge to the true values and the amounts of the posterior risks tend to decrease by increasing the sample size. This simply indicates that the proposed estimators are consistent. The smaller (larger) values of the parameter representing one component of the mixture impose a positive (negative) impact on the estimation of the parameter representing the other component of the mixture distribution. The larger values of the mixing parameter $\left(p_{1}\right)$ impose a positive impact on the performance of the estimation of the first component of the mixture. This may be due to the reason that the lager values of the mixing parameter incorporate more values for the analysis of the first component. Bayes estimators performed well under the precautionary loss function than the weighted squared error loss function under both priors. In addition, the performance of the estimates under gamma prior is better than those under inverse 
levy prior using both loss functions. However, in case of gamma prior the estimates under both loss functions are comparatively more under estimated, though this problem is less severe in the larger samples. Therefore on the basis of the above discussion we can recommend the use of gamma prior under precautionary loss function for the analysis of the inverse weibull mixture distribution under the Bayesian framework.

However, when we use such a mixture model in real-life we can chose the prior as well as the loss function according to the need. In case of loss functions, if lower posterior risk is desired than in the present scenario, the precautionary loss function should be given importance, if compromise on risk is affordable than one can easily select to use the kloss function. Also the informative gamma prior can easily be preferred over the other informative prior as shown by results. It may be mentioned here that because of space restriction, only selected results are included and presented graphically. The findings of real life example are in accordance with the simulation study. The findings of the paper are useful for the analysts (from different fields) dealing with the Bayesian analysis of the time to failure data when causes of the failure are more than one, and the data is doubly censored.

\section{Reference}

1. Aslam, M. (2003). An application of prior predictive distribution to elicit the prior density, Journal of Statistical Theory and Applications. 2(1), 70-83.

2. Bekker, A., Roux, J. and Mostert, P. (2000). A generalization of the compound Rayleigh distribution: using a Bayesian method on cancer survival times. Communications in Statistics, Theory and Methods, 29(7), 1419-1433.

3. Bolstad, W. M. (2004). Introduction to Bayesian Statistics. John Wiley and Sons, Inc. New York.

4. Kundu, D. and Howlader H. (2010). Bayesian inference and prediction of the inverse Weibull distribution for type-II censored data, Comput. Stat. Data Anal. 54(6), 1547-1558.

5. Panaitescu, E., George, P., Cozma, P. and Popa, M. (2010). Bayesian and nonBayesian estimators using record statistics of the modified-inverse Weibull distribution, Proc. Rom. Academy Ser. A, 11(3), 224-231.

6. Eluebaly, T. and Bougila, N. (2011). Bayesian learning of finite generalized Gaussian mixture models on images. Signal Processing, 91 (4), 801-820.

7. Fernandez, A. J. (2000). On maximum likelihood prediction based on type II doubly censored exponential data. Metrika, 50(3), 211-220.

8. Fernandez, A. J. (2006). Bayesian estimation based on trimmed samples from Pareto populations. Computational statistics \& data analysis, 51(2), 1119-1130.

9. Ghosh, S. K. and Ebrahimi, N. (2001). Bayesian analysis of the mixing function in a mixture of two exponential distributions. Tech. Rep. 2531, Institute of Statistics Mimeographs, North Carolina State University, North Carolina State University. 
10. Grimshaw, S. D., Collings, B. J., Larsen, W. A. and Hurt, C. R. (2001). Eliciting Factor Importance in a Designed Experiment. Technometrics, 43(2), 133-146.

11. Jenkinson, D. (2005). The elicitation of probabilities: A review of the statistical literature, Department of Probability and Statistics, University of Sheffield.

12. Sultan, K. S., Ismail, M. A. and Al-Moisheer, A. S. (2007). Mixture of two inverse Weibull distributions: properties and estimation, Comput. Stat. Data Anal. 51, 5377-5387.

13. Khan, H. M., Albatineh, A., Alshahrani, S., Jenkins, N. and Ahmed, N. U. (2011). Sensitivity analysis of predictive modeling for responses from the three-parameter Weibull model with a follow-up doubly censored sample of cancer patients. Computational Statistics \& Data Analysis, 55(12), 3093-3103.

14. Khan, H. M., Provost, S. B. and Singh, A. (2010). Predictive inference from a two-parameter Rayleigh life model given a doubly censored sample. Communications in Statistics - Theory and Methods, 39(7), 1237-1246.

15. Kim, C. and Song, S. (2010). Bayesian estimation of the parameters of the generalized exponential distribution from doubly censored samples. Statistical Papers, 51(3), 583-597.

16. Kundu, D. and Howlader, H. (2010). Bayesian inference and prediction of the inverse Weibull distribution for type-II censored data, Comput. Stat. Data Anal. 54(6), 1547-1558.

17. Leon, J. C., Vazquez-Polo, J. F. and Gonzalez, L.R. (2003). Environmental and Resource Economics, Kluwer Academic Publishers, 26, 199-210

18. Mendenhall, W. and Hadar, R. J. (1958). Estimation of parameters of mixed exponentially distributed failure time distributions from censored life test data. Biometrika, 45, 504-520.

19. Murthy, D. N. P., Bulmer, M. and Eccleston, J. A. (2004). Weibull model selection for reliability modelling, Reliab. Eng. Syst. Saf. 86, 257-267.

20. Noor, F. and Aslam, M. (2008). Bayesian inference of the inverse Weibull mixture distribution using type-I censoring. Journal of Applied Statistics, 40(5), 1076-1089.

21. O'Hagan, A., Buck, C. E., Daneshkhah, A., Eiser, J. E., Garthwaite, P. H., Jenkinson, D. J., Oakley, J. E. and Rakow, T. (2006). Uncertain Judgements: Eliciting expert probabilities. John Wiley \& Sons.

22. Pak, A., Parham, G. A. and Saraj, M. (2013). On Estimation of Rayleigh Scale Parameter under Doubly Type-II Censoring from Imprecise Data. Journal of Data Science, 11, 305-322.

23. Zanakis, S. H. (2007). A simulation study of some simple estimators for the threeparameter Weibull distribution, J. Stat. Comput. Simul. 9, 101-116.

24. Saleem, M. and Aslam, M. (2008). Bayesian analysis of the two component mixture of the Rayleigh distribution assuming the Uniform and the Jeffreys prior from censored data, J. App. Statist. Science, 16(4), 105-113. 
25. Saleem, M., Aslam, M. and Economou, P. (2010). On the Bayesian analysis of the mixture of Power function distribution using the complete and the censored sample. J. Applied Statistics, 37(1), 25-40.

26. Shi, Y. and Yan, W. (2010). The EB estimation of scale parameter for two parameter exponential distribution under the type-I censoring life test, J. Phys. Sci. 4, 25-30.

27. Singh, S. K., Singh, U. and Sharma, V. K. (2013). Bayesian analysis for Type-II hybrid censored sample from inverse Weibull distribution. Int. J. Assure. Eng. Manag., 1-8.

28. Singh, S. K., Umesh S., and Vikas K. S. (2013). Bayesian analysis for Type-II hybrid censored sample from inverse Weibull distribution. International Journal of System Assurance Engineering and Management: 1-8.

29. Soliman (2006). Estimators for the finite mixture of Rayleigh model based on progressively censored data. Communications in Statistics-Theory and Methods, 35(5), 803-820.

30. Sultan, K. S. and Al-Moisheer, A. S. (2012). Approximate Bayes estimation of the parameters and reliability function of a mixture of two inverse Weibull distributions under type-2 censoring. Journal of Statistical Computation and Simulation, 83(10), 1900-1914.

31. Sultan, K. S., Ismail, M. A. and Al-Moisheer, A. S. (2007). Mixture of two inverse Weibull distributions: Properties and estimation. Computational Statistics \& Data Analysis, 51(11), 5377-5387.

32. Tummala, V. M. and Sathe, P. T. (1978). Minimum expected loss functions of reliability and parameters of certain lifetime distributions. IEEE Trans. Reliability, 27(4), 283-285

33. Blischke, W. R. and Murthy, D. N. P. (2000). Reliability, Wiley, New York.

34. Wasan, M. T. (1970). Parametric Estimation, McGraw-Hill Book Company, New York.

35. Shi, Y. and Yan, W. (2010). The EB estimation of scale parameter for two parameter exponential distribution under the type-I censoring life test, J. Phys. Sci. 4, 25-30. 